\title{
A Retrospective Comparison of the Modified Kakita Method and the Modified Cattel-Warren Anastomosis
}

\author{
Fumiyoshi Saito $^{1 *}$, Kenichiro Araki ${ }^{1}$, Norio Kubo ${ }^{1}$, Akira Watanabe ${ }^{1}$, Mariko Tsukagoshi ${ }^{1}$, Norihiro Ishii ${ }^{1}$, Ken Shirabe ${ }^{2}$ and \\ Hiroyuki Kuwano ${ }^{1}$ \\ ${ }^{1}$ Department of General Surgical Science, Gunma University, Graduate School of Medicine, Gunma 371-8511, Japan \\ ${ }^{2}$ Department of Hepato-Biliary-Pancreatic surgery, Gunma University, Graduate School of Medicine, Gunma 371-8511, Japan
}

${ }^{*}$ Corresponding author: Fumiyoshi Saito, Department of General Surgical Science, Gunma University, Graduate School of Medicine, 3-39-22 Syouwamachi, Maebashi, Gunma 371-8511, Japan; E-mail: saito_6530@mac.com

Received: December 30, 2020; Accepted: January 06, 2021; Published: January 07, 2020

\begin{abstract}
Objective: This study compared the perioperative outcomes from patients who underwent pancreaticojejunostomy in pancreaticoduodenectomy via the modified Kakita method anastomosis (KMA) or the modified Cattell-Warren anastomosis (CWA).

Summary of background data: We retrospectively evaluated 43 consecutive patients who underwent pancreaticoduodenectomy between January 2006 and December 2012.

Methods: The modified CWA was exclusively performed before December 2009, and the modified KMA was exclusively performed after January 2010. To evaluate their simplicity and safety, we compared the perioperative outcomes for the patients who underwent CWA $(n=22)$ and the patients who underwent KMA $(\mathrm{n}=21)$.
\end{abstract}

Results: Pancreatic fistula was significantly less frequent in the KMA group, compared to in the CWA group ( $4.8 \%$ vs. $36.3 \%$ respectively, $p=0.021)$. In addition, the rate of all surgical complications decreased after the introduction of KMA at our institution.

Conclusions: The results of this retrospective study appear to indicate that KMA is a simpler and safer technique, compared to CWA, for pancreaticojejunostomy in pancreaticoduodenectomy.

Keywords: Kakita method anastomosis, Pancreaticoduodenectomy, Pancreaticojejunostomy, Pancreatic fistula

\section{Manuscript Summary}

The major finding(s) from the study: Our results indicate that the modified Kakita technique provided a significantly lower frequency of pancreatic fistula, as well as non-significant reductions in other postoperative complications.

What the findings add to existing knowledge: We conclude that the modified Kakita technique may be simpler and more effective than the modified Cattel-Warren technique.

What is already known in the field: $\mathrm{U}$-sutures may reduce shear forces at the fragile pancreatic parenchyma, and subsequently reduce the incidence of pancreatic fistula.

What should change as a result: We will perform the modified Kakita method anastomosis.

\section{Introduction}

The history of pancreaticojejunostomy in pancreaticoduodenectomy has been described throughout the literature, with Whipple et al. reporting the first cases of pancreaticoduodenectomy in 1935 [1]. Whipple also introduced pancreaticojejunostomy with complete one-stage reconstruction in 1946 [2]. However, in 1943, Cattell stated that pancreaticoenteric anastomosis was indispensable, and maintained that leakage of the pancreatic juice accounted for many postoperative complications and deaths among patients who underwent pancreaticoduodenectomy [3]. Therefore, Cattell recommended direct anastomosis of the pancreatic duct and jejunum in patients with a main pancreatic duct that had a sufficient diameter. However, for smaller pancreatic ducts, Cattell recommended the use of a "necrosing suture", whereby the pancreatic duct was ligated and the cut surface of the pancreas was covered with the jejunal wall. Unfortunately, pancreaticoduodenectomy has historically had high rates of complications and operative mortality, which were often related to suture failure during pancreaticojejunostomy in pancreaticoduodenectomy. However, some high-volume institutions have reported mortality rates of $<5 \%$ for pancreaticoduodenectomy [4-7], although the postoperative morbidity rates remain high, ranging from $30 \%$ to $50 \%$ [6-13].

Pancreatic fistula is a well-known complication of pancreaticoduodenectomy, with rates of $2-20 \%$ being recently reported [7-17]. To address this issue, several different anastomotic techniques have been used to minimize the incidence of pancreatic 
fistula $[15,16]$. These techniques include the modified Cattell-Warren anastomosis (CWA) 3, Peng's method [16], Blumgart's method [17], invaginating the pancreatic stump into the jejunal stump [18], and the modified Kakita method anastomosis (KMA) [19,20]. In this retrospective study, we compared the perioperative outcomes for pancreaticoduodenectomy among patients who underwent the CWA and KMA procedures.

\section{Metholds}

\section{Patients}

Between January 2006 and December 2012, 43 consecutive patients underwent pancreaticoduodenectomy with pancreaticojejunostomy in the Department of Gastroenterological Surgery at Tomei Atugi Hospital, and were entered into our prospective database. The modified CWA method was exclusively performed before December 2009, and the modified KMA method was exclusively performed after January 2010. Using a before-after cohort design, we compared the perioperative outcomes for the CWA $(\mathrm{n}=22)$ and the KMA $(\mathrm{n}=21)$ groups.

\section{Surgical Technique and Postoperative Management}

All surgical procedures were performed by or under the supervision of experienced pancreatic surgeons. Most patients underwent subtotal stomach-preserving pancreaticoduodenectomy (SSpP), which involves resection of the pyloric ring and preservation of $>95 \%$ of the stomach, although some patients underwent conventional pancreaticoduodenectomy with distal gastrectomy. Reconstruction was performed using a modified Child's technique for both SSpPD and conventional pancreaticoduodenectomy. The anastomosis was performed (in order of preference) between the jejunum and pancreas, bile duct, and stomach. Drain tubes (8-mm silicone tubes) ware placed at the ventral and dorsal sides of the pancreaticojejunostomy. Oral fluids were started at $72 \mathrm{~h}$ after the surgery, and oral intake was started at approximately 5 days after surgery, except in cases with postoperative complications, such as delayed gastric emptying. All abdominal drains were removed at day 7 after the surgery if the drainage fluid was clear, did not exceed $300 \mathrm{~mL}$ per $24 \mathrm{~h}$, and contained a concentration of amylase that was $<3$-fold greater than the serum concentration. Second-generation cephem antibiotics were administered immediately before surgery and every $3 \mathrm{~h}$ during surgery, with continuation until day 3 after the surgery. In cases that contracted an infectious disease, the antibiotics were changes as necessary; octreotide was not routinely used.

\section{The Modified KMA Technique}

The pancreatic duct and jejunal mucosa were joined in an end-toside fashion, using eight absorbable interrupted sutures (PDSII 5/0, ETHICON) via the duct-to-mucosa anastomosis. All patients who underwent KMA had a 4-Fr to 6-Fr polyvinyl catheter inserted into the main pancreatic duct for external drainage. The unique aspect of this modified KMA technique is the approximation of the pancreatic parenchyma to the jejunal seromuscular layer, using five or six nonabsorbable interrupted penetrating sutures (Prolene 3/0, ETHICON) $[19,20]$.

\section{The Modified CWA Technique}

The modified CWA was performed after a small incision was made at the antimesenteric side of the jejunal loop. Monofilament absorbable interrupted sutures (PDSII 3/0, ETHICON) were placed using an atraumatic needle, beginning at the posterior surface of the pancreas. The dorsal capsule of the pancreas was sutured to the seromuscular layer of the jejunum, and then the central portion of the anastomosis was completed as a duct-to-mucosa anastomosis, using interrupted sutures (PDSII 5/0, ETHICON). Finally, monofilament absorbable interrupted sutures (PDSII 3/0, ETHICON) were placed at the anterior surface of the pancreas [3]. All patients who underwent CWA had a 4-Fr to 6-Fr polyvinyl catheter inserted into the main pancreatic duct for external drainage.

\section{Data Collection and Evaluation Parameters}

We retrospectively reviewed our institution's database to obtain the following case-specific information: age, sex, preoperative biliary drainage, diagnosis, medical history, preoperative laboratory findings (serum glutamic oxaloacetic transaminase, bilirubin, alkaline phosphatase, albumin, creatinine, lipase, amylase, hemoglobin, white cell count, C-reactive protein, and partial thromboplastin time), body mass index, pancreatic texture, operative time, intraoperative blood loss, number and type of postoperative local and systemic complications, and mortality. Postoperative morbidity was defined as any postoperative surgical or non-surgical complication. Postoperative pancreatic fistula (POPF) was diagnosed and graded based on the International Study Group on Pancreatic Fistula guidelines. The allinclusive definition was a drain output of any measurable fluid volume on or after postoperative day 3 , with amylase concentration of $>3$-fold higher than the serum amylase concentration. Three different grades of POPF (grades A, B, C) were defined according to the clinical signs of infection and/or a necessary change in the clinical management [21]. A fistula of grade B (fistula requiring any therapeutic intervention) or higher was considered clinically significant.

\section{Statistical Analysis}

Consecutive data were expressed as median (range) and were analyzed using the Mann-Whitney $\mathrm{U}$ test. Inter-group differences in numerical data were evaluated using the $\chi^{2}$ test (with Yates correction) or Fisher's exact test when the n-value was $<5$. All statistical analyses were performed using Ystat2013 (Microsoft Excel), and differences with a p-value of $<0.05$ were considered statistically significant.

\section{Results}

\section{Patient Characteristics}

This study evaluated 22 patients who underwent CWA and 21 patients who underwent KMA; their characteristics are shown in Table 1. However, there were no significant differences in age or sex when we compared the two groups. In the CWA group, the pathological diagnoses were pancreatic cancer in 10 patients, cholangiocarcinoma in 9 patients, and intraductal papillary mucinous neoplasms in 3 patients. In the KMA group, the pathological diagnoses were pancreatic cancer in 10 patients, cholangiocarcinoma in 9 patients, and cystic intraduct- 
Table 1: Patient characteristics.

\begin{tabular}{|c|c|c|c|}
\hline & Modified Cattell-Warren anastomosis $(\mathrm{n}=\mathbf{2 2})$ & Modified Kakita method anastomosis $(\mathrm{n}=\mathbf{2 1})$ & p-value \\
\hline Age (years) & $69(56-86)$ & $65(32-84)$ & 0.518 \\
\hline Sex (male/female) & 16.06 & 14.07 & 0.92 \\
\hline \multicolumn{4}{|l|}{ Diagnosis } \\
\hline Pancreatic cancer & 10 & 10 & 0.886 \\
\hline IPMN* $^{*}$ & 3 & 2 & 1 \\
\hline Cholangiocarcinoma & 9 & 9 & 0.857 \\
\hline \multicolumn{4}{|l|}{ Pancreatic texture } \\
\hline Hard pancreas & 9 & 10 & 0.892 \\
\hline Soft pancreas & 13 & 11 & 0.892 \\
\hline Duration of operation (min) & 580 & 520 & 0.345 \\
\hline Estimated blood loss (mL) & 978 & 933 & 0.5 \\
\hline IPMN: intraductal papillary $\mathrm{m}$ & & & \\
\hline
\end{tabular}

Table 2: Postoperative complication.

\begin{tabular}{|c|c|c|c|}
\hline & Modified Cattell-Warren anastomosis $(n=22)$ & Modified Kakita method anastomosis $(\mathrm{n}=21)$ & p-value \\
\hline Surgical complications & $10(45.4 \%)$ & $5(23.8 \%)$ & 0.242 \\
\hline Wound infection & $2(9.0 \%)$ & $3(14.4 \%)$ & 0.664 \\
\hline Intra-abdominal abscess & $4(18.2 \%)$ & $1(4.8 \%)$ & 0.344 \\
\hline Chylous ascites & $1(4.5 \%)$ & $1(4.8 \%)$ & 1 \\
\hline Anastomotic hemorrhage & $1(4.5 \%)$ & 0 & 1 \\
\hline Delayed gastric emptying & $2(9.1 \%)$ & $1(4.8 \%)$ & 1 \\
\hline Hemorrhage of pseudoaneurysm & $1(4.5 \%)$ & 0 & 1 \\
\hline Pancreatic fistula & $8(36.3 \%)$ & $1(4.8 \%)$ & 0.021 \\
\hline Non-surgical complications & $2(9.0 \%)$ & $5(23.8 \%)$ & 0.24 \\
\hline Enteritis & $1(4.5 \%)$ & $2(9.5 \%)$ & 0.606 \\
\hline Deep venous thrombosis & 0 & $1(4.8 \%)$ & 0.488 \\
\hline Respiratory events & 0 & $3(14.4 \%)$ & 0.107 \\
\hline Catheter-associated infections & $1(4.5 \%)$ & $1(4.8 \%)$ & 1 \\
\hline Total surgical and non-surgical complications & $12(54.6 \%)$ & $10(47.6 \%)$ & 0.649 \\
\hline Mortality & 0 & 0 & \\
\hline
\end{tabular}

al papillary mucinous neoplasm in 2 patients. When we compared the two groups, no significant differences were observed for pancreatic texture (hard/soft), mean operative time, or intraoperative blood loss.

\section{Postoperative Complications}

The types and frequencies of the postoperative complications are shown in Table 2. Pancreatic fistula occurred significantly less frequently in the KMA group, compared to in the CWA group (4.8\% vs. $36.3 \%, p=0.021$ ), and one case of pancreatic fistula-related hemorrhage was observed in the CWA group. When we compared the specific incidences of pancreatic fistulas, grade B or C fistula was recognized in one case for the KMA group, compared to 7 cases for the CWA group, with latent presentation of a pancreatic fistula in one case. In the case with latent presentation of the pancreatic fistula, the drainage fluid amylase concentration was not elevated during the postoperative period, although the fistula was diagnosed via computed tomography after the drain was removed (Table 3). In addition, we observed a noticeable, although not significant, difference in the frequency of surgical complications after the introduction of KMA (23.8\% after KMA vs. $45 \%$ after CWA; $\mathrm{p}=0.242)$. Furthermore, the KMA group experienced fewer morbidities, although this difference was also not statistically significant $(52.3 \%$ vs. $68.1 \%, p=0.597)$. No cases of in-hospital mortality were observed for either group.

\section{Comparing the Drainage Fluid Amylase Concentrations and Duration of Drain Insertion}

When we compared the two groups, no significant differences were observed in the median drainage fluid amylase concentration in the CWA and KMA groups (CWA: $98 \mathrm{IU} / \mathrm{L}$; range, 2-83,900 IU/L; KMA: $45 \mathrm{IU} / \mathrm{L}$; range, 6-1,036 IU/L) (Figure 1). The drainage fluid amylase concentration exceeded 1,000 IU/L in 4 cases ( 3 cases in the CWA group and one case in the KMA group) on or after postoperative 
Table 3: Comparison of pancreatic fistula incidence for Cattell-Warren and Kakita method anastomosis.

\begin{tabular}{|l|c|c|c|}
\hline & Modified Cattell-Warren anastomosis (n= 22) & Modified Kakita method anastomosis (n= 21) & p-value \\
\hline No pancreatic fistula or Grade A & 14 & 20 & 0.0448 \\
\hline Grade B and Grade C & 7 & 1 & 0.0448 \\
\hline Latent pancreatic fistula & 1 & 0 & 1 \\
\hline Pancreatic fistula & 8 & 1 & 0.0212 \\
\hline
\end{tabular}

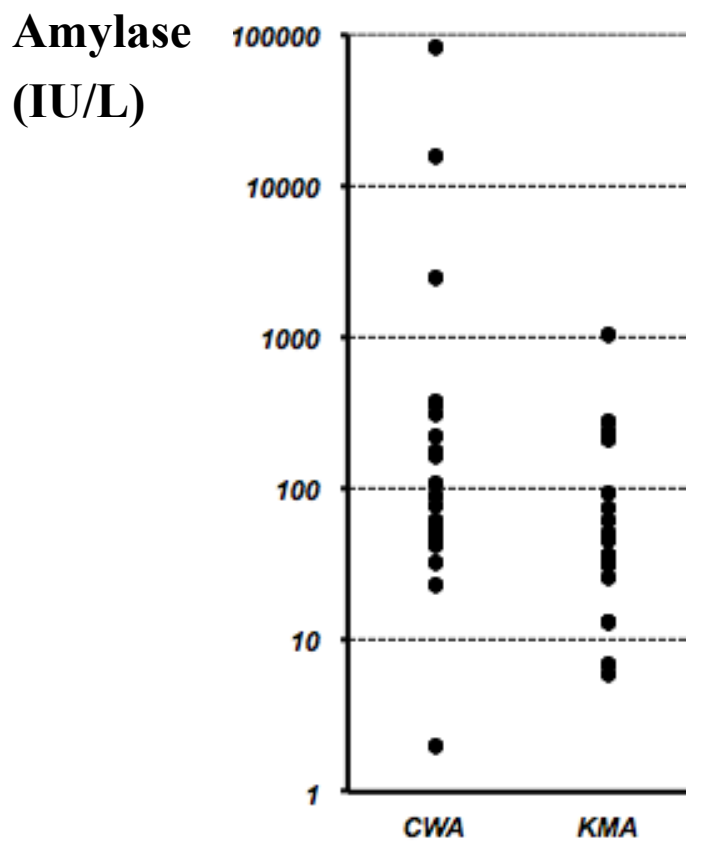

Figure 1: Amylase concentrations in the drainage fluid for all cases.

No significant difference was observed when we compared the median amylase concentrations in the drainage fluids from the Cattell-Warren anastomosis (CWA) group (median, $98 \mathrm{IU} / \mathrm{L}$; range, 2-83,900 IU/L) and the Kakita method anastomosis (KMA) group (median, $45 \mathrm{IU} / \mathrm{L}$; range, $6-1,036 \mathrm{IU} / \mathrm{L}$ ). $\mathrm{P}=0.088$ via the Mann-Whitney U-test.

day 3. When we compared the duration of drain insertion for both groups, no significant difference in the median duration was observed (CWA: 16 days; range, 7-94 days; KMA: 14 days; range, 7-57 days) (Figure 2).

\section{Discussion}

The techniques that are used for reconstruction of the pancreatic stump after pancreaticoduodenectomy are closely related to the incidence of postoperative complications, mortality, and reduced quality of life. Pancreatic fistula is a well-known complication of pancreaticoduodenectomy, with rates of 2-20\% being recently reported $[7,8,10,12,14-17,20,22,23]$. In many institutions, several different surgical procedures, such as Blumgart anastomosis, have been used to minimize the incidence of pancreatic fistula. Among these procedures, CWA is the most well-known procedure, and has been commonly used for a long period of time. In contrast, KMA is a relatively simple technique, and many surgeons in Japan perform KMA in pancreasjejunum anastomosis. In this retrospective study, we found that KMA appeared to be a simpler and safer technique for pancreaticojejunostomy, compared to CWA. Moreover, the KMA technique significantly reduced the frequency of pancreatic fistula, with non-statistically significant reductions for other postoperative complications.

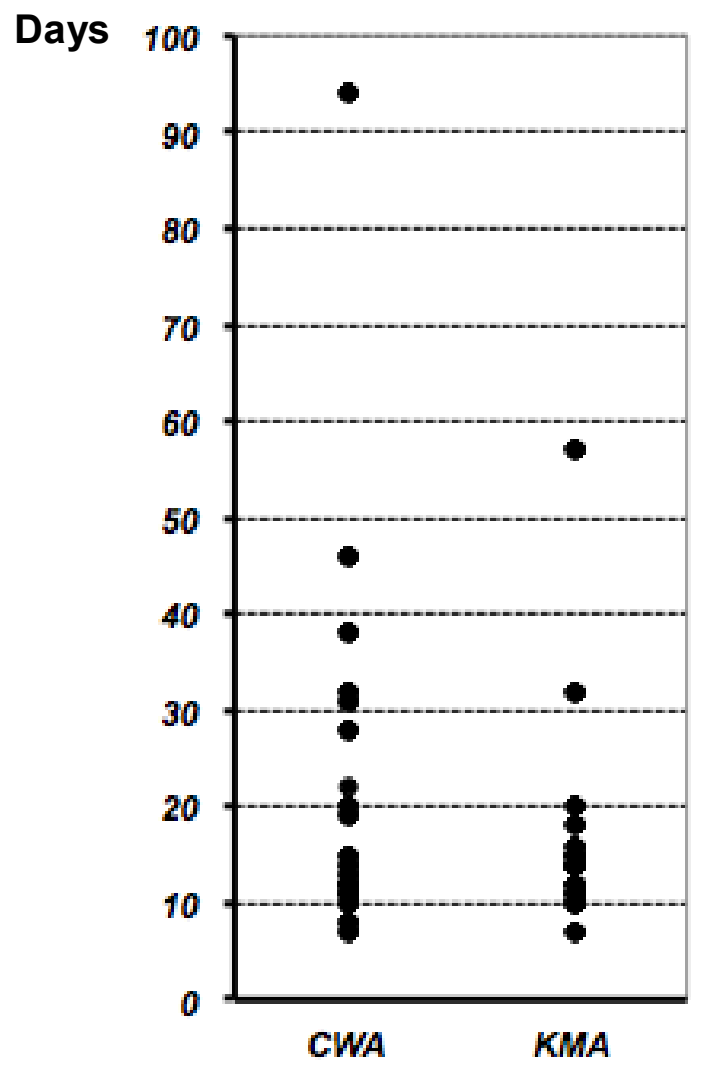

Figure 2: Duration of drain insertion for all cases.

No significant difference was observed when we compared the median duration of drain insertion for the Cattell-Warren anastomosis (CWA) group (median, 16 days; range, 7-94 days) and the Kakita method anastomosis (KMA) group (median, 14 days; range, 7-57 days). $\mathrm{P}=0.501$ via the Mann-Whitney U-test.

Patient age and intraoperative blood loss have been identified as perioperative risk factors for pancreatic fistula. In addition, soft pancreatic texture, pancreatic duct size, and pancreatic juice output have been reported to be predictive factors for pancreatic fistula $[24,25]$. In the present study, we observed similar trends within both groups, although there were no significant differences when we compared the risk and predictive factors between the two groups.

In CWA, multiple sutures are placed tangentially through the pancreatic capsule, which may create shear forces at the fragile pancreatic parenchyma. Furthermore, the knot-tying may cause the sutures to cut through the pancreas, and the use of multiple sutures is known to cause pancreatic microleakage during the knot-tying [17]. Therefore, it has been speculated that the use of too many sutures and/or too aggressive knot-tying may cause ischemia and necrosis of the pancreatic stump. In contrast, KMA uses only five or six non-absorbable interrupted penetrating sutures to approximate 
the pancreatic parenchyma to the jejunal seromuscular layer. Thus, this technique reduces the total number of sutures, avoids placing unnecessary shear forces on the fragile pancreatic parenchyma, and avoids some of the complicated manipulations that are required for other surgical techniques [19]. Furthermore, the KMA technique can help to reduce the risk of suture failure as a result of necrosis and ischemia.

Various previous studies have compared different anastomosis techniques, such as pancreaticojejunostomy versus pancreaticogastrostomy [25], Blumgart anastomosis versus modified CWA [17] or versus the Kakita type anastomosis [26], pancreaticojejunostomy with the invagination technique (dunking) versus duct-to-mucosa pancreaticojejunostomy [27], or binding anastomosis [28]. In addition, prospective randomized trials have compared pancreaticojejunostomy to pancreaticogastrostomy, and found that both procedures provided similar incidences of pancreatic fistula $[25,29,30]$. Similarly, a comparison of the invagination method and pancreatic duct jejunum anastomosis found no difference in the incidence of pancreatic fistula [6]. However, Blumgart anastomosis was associated with a lower incidence of pancreatic fistula, compared to the modified CWA ( $4 \%$ vs. $13 \%$, respectively) [17] or to Kakita type anastomosis ( $2.5 \%$ vs. $36 \%$, respectively) [26]. Similarly, the recessed method has been reported to provide a low incidence of pancreatic fistula [27], and Peng et al. have reported pancreatic leakage rates of $0 \%$ using a complex three-layer dunking anastomosis [29,31,32], although this procedure is technically difficult. Interestingly, the Blumgart and "dunking" invagination techniques use U-sutures $[33,34]$, and these techniques provide relatively low complication rates. Therefore, U-sutures may reduce shear forces at the fragile pancreatic parenchyma, and subsequently reduce the incidence of pancreatic fistula. Similarly, the KMA method attempts to reduce the shear force in a manner that is similar to that performed with U-sutures.

Unfortunately, despite various techniques having been developed to manage the pancreatic remnant after pancreaticoduodenectomy, none of these techniques are associated with clearly superior outcomes. Thus, it is important to preserve the pancreatic capsule and to avoid bleeding from the pancreatic parenchyma during pancreaticojejunostomy, which can affect hemostasis in that tissue. Therefore, it is important to use surgical and suturing techniques that preserves as much of the parenchyma as possible (by not placing unnecessary shearing force on the pancreas).

This study has several limitations. First, because it is a retrospective single-center study, there are limitations regarding the generalizability of our data. In addition, over the course of 6 years, there is a possibility that the postoperative management may have changed slightly. Furthermore, it is impossible to completely exclude the potential effect of confounders (e.g., surgical standards and perioperative management), although it is unlikely that these factors strongly influenced the incidence of pancreatic fistula and suture insufficiency. Nevertheless, our results indicate that KMA was a simple and safe technique for reducing the incidence of pancreatic fistula and leakage rates after pancreaticojejunostomy.

\section{References}

1. Whipple AO, Parsons WB, Mullins CR (1935) TREATMENT OF CARCINOMA OF THE AMPULLA OF VATER. Ann Surg 102(4): $763-779$ [crossref]

2. Whipple AO (1946) Observations on radical surgery for lesions of the pancreas. Surg Gynecol Obstet 82: 623-631 [crossref]

3. Cattell RB (1949) The surgical treatment of carcinoma of the pancreatoduodenal area. Ann R Coll Surg Engl 4(4): 197-205 [crossref]

4. Poon RT, Lo SH, Fong D, Fan ST, Wong J (2002) Prevention of pancreatic anastomotic leakage after pancreaticoduodenectomy. Am J Surg 183(1): 42-52_crossref]

5. Shapiro TM (1975) Adenocarcinoma of the pancreas: a statistical analysis of biliary bypass vs Whipple resection in good risk patients. Ann Surg 182(6): 715-721 [crossref]

6. Bassi C, Falconi M, Molinari E, Mantovani W, Butturini G. et al. (2003) Ductto-mucosa versus end-to-side pancreaticojejunostomy reconstruction after pancreaticoduodenectomy: results of a prospective randomized trial. Surgery 134(5): 766-771 [crossref]

7. Buchler MW, Friess H, Wagner M, Kulli C, Wagener V. et al. (2000) Pancreatic fistula after pancreatic head resection. Br J Surg 87(7): 883-889 [crossref]

8. Takano S, Ito Y, Watanabe Y, Yokoyama T, Kubota N. et al. (2000) Pancreaticojejunostomy versus pancreaticogastrostomy in reconstruction following pancreaticoduodenectomy. Br J Surg 87(4): 423-427 [crossref]

9. Birkmeyer JD, Siewers AE, Finlayson EV, Stukel TA, Lucas FL (2002) et al. Hospital volume and surgical mortality in the United States. N Engl J Med 346(15): 1128-1137 [crossref]

10. Yeo CJ, Cameron JL, Lillemoe KD, Sauter PK, Coleman J. et al. (2000) Does prophylactic octreotide decrease the rates of pancreatic fistula and other complications after pancreaticoduodenectomy? Results of a prospective randomized placebo-controlled trial. Ann Surg 232(3): 419-429 [crossref]

11. Grobmyer SR, Rivadeneira DE, Goodman CA, Mackrell P, Lieberman MD (2000) Pancreatic anastomotic failure after pancreaticoduodenectomy. Am J Surg 180(2): $117-120$ [crossref]

12. Ohwada S, Ogawa T, Kawate S, Tanahashi Y, Iwazaki S. et al. (2001) Results of ductto-mucosa pancreaticojejunostomy for pancreaticoduodenectomy Billroth I type reconstruction in 100 consecutive patients. J Am Coll Surg 193(1): 29-35 [crossref]

13. Shyr YM, Su CH, Wu CW, Lui WY (2003) Does drainage fluid amylase reflect pancreatic leakage after pancreaticoduodenectomy? World J Surg 27(5): 606-610 [crossref]

14. Strasberg SM, Drebin JA, Mokadam NA, Green DW, Jones KL, et al. (2002) Prospective trial of a blood supply-based technique of pancreaticojejunostomy: effect on anastomotic failure in the Whipple procedure. J Am Coll Surg 194(6): 746-758; discussion 759-760 [crossref]

15. Rault A, SaCunha A, Klopfenstein D, Larroude D, Epoy FN, et al. (2005) Pancreaticojejunal anastomosis is preferable to pancreaticogastrostomy after pancreaticoduodenectomy for longterm outcomes of pancreatic exocrine function. J Am Coll Surg 201(2): 239-244 [crossref]

16. Peng SY, Wang JW, Li JT, Mou YP, Liu YB, et al. (2004) Binding pancreaticojejunostomy-a safe and reliable anastomosis procedure. HPB (Oxford) 6(3): 154-160 [crossref]

17. Kleespies A, Rentsch M, Seeliger H, Albertsmeier M, Jauch KW. et al. (2009) Blumgart anastomosis for pancreaticojejunostomy minimizes severe complications after pancreatic head resection. Br J Surg 96(7): 741-750 [crossref]

18. Bugiantella W, Rondelli F, Mariani L, Longaroni M, Federici MT, et al. (2014) Pancreatico-jejunal anastomosis with invaginating jenunal "J"-loop. Preliminary report of a new technique. Int J Surg 12 Suppl 1: S87-90

19. Kakita A, Yoshida M, Takahashi T (2001) History of pancreaticojejunostomy in pancreaticoduodenectomy: development of a more reliable anastomosis technique. J Hepatobiliary Pancreat Surg 8(3): 230-237

20. Kakita A, Takahashi T, Yoshida M, Furuta K (1996) A simpler and more reliable technique of pancreatojejunal anastomosis. Surg Today 26(7): 532-535 [crossref]

21. Bassi C, Dervenis C, Butturini G, Fingerhut A, Yeo C, et al. (2005) Postoperative pancreatic fistula: an international study group (ISGPF) definition. Surgery 138(1): 8-13 [crossref] 
22. Kanda M, Fujii T, Kodera Y, Nagai S, Takeda S, et al. (2011) Nutritional predictors of postoperative outcome in pancreatic cancer. BrJ Surg 98(2): 268-274 [crossref]

23. Lai EC, Lau SH, Lau WY (2009) Measures to prevent pancreatic fistula after pancreatoduodenectomy: a comprehensive review. Arch Surg 144(11): 1074-1080 [crossref]

24. Kollmar O, Moussavian MR, Bolli M, Richter S, Schilling MK (2007) Pancreatojejunal leakage after pancreas head resection: anatomic and surgeon-related factors. J Gastrointest Surg 11(12): 1699-1703 [crossref]

25. Bassi C, Falconi M, Molinari E, Salvia R, Butturini G, et al. (2005) Reconstruction by pancreaticojejunostomy versus pancreaticogastrostomy following pancreatectomy: results of a comparative study. Ann Surg 242(6): 767-771, discussion 771-763 [crossref]

26. Fujii T, Sugimoto H, Yamada S, Kanda M, Suenaga M et al. (2014) Modified Blumgart anastomosis for pancreaticojejunostomy: technical improvement in matched historical control study. J Gastrointest Surg 18(6): 1108-1115 [crossref]

27. Berger AC, Howard TJ, Kennedy EP, Sauter PK, Bower-Cherry M, et al. (2009) Does type of pancreaticojejunostomy after pancreaticoduodenectomy decrease rate of pancreatic fistula? A randomized, prospective, dual-institution trial. J Am Coll Surg 208(5): 738-747; discussion 747-739 [crossref]
28. Peng S, Mou Y, Cai X, Peng C (2002) Binding pancreaticojejunostomy is a new technique to minimize leakage. Am J Surg 183(3): 283-285 [crossref]

29. Yeo CJ, Cameron JL, Maher MM, Sauter PK, Zahurak ML, et al. (1995) A prospective randomized trial of pancreaticogastrostomy versus pancreaticojejunostomy after pancreaticoduodenectomy. Ann Surg 222(4): 580-588; discussion 588-592 [crossref]

30. Duffas JP, Suc B, Msika S, Fourtanier G, Muscari F, et al. (2005) A controlled randomized multicenter trial of pancreatogastrostomy or pancreatojejunostomy after pancreatoduodenectomy. Am J Surg 189(6): 720-729 [crossref]

31. Peng SY, Mou YP, Liu YB, Su Y, Peng CH, et al. (2003) Binding pancreaticojejunostomy: 150 consecutive cases without leakage. J Gastrointest Surg 7(7): 898-900 [crossref]

32. Peng SY, Wang JW, Lau WY, Cai XJ, Mou YP, et al. (2007) Conventional versus binding pancreaticojejunostomy after pancreaticoduodenectomy: a prospective randomized trial. Ann Surg 245(5): 692-698 [crossref]

33. Chen XP, Huang ZY, Lau JW, Zhang BX, Zhang ZW, et al. (2014) Chen's U-suture technique for end-to-end invaginated pancreaticojejunostomy following pancreaticoduodenectomy. Ann Surg Oncol 21(13): 4336-4341 [crossref]

34. Kennedy EP, Yeo CJ. (2011) Dunking pancreaticojejunostomy versus duct-to-mucosa anastomosis. J Hepatobiliary Pancreat Sci 18(6): 769-774 [crossref] 